\title{
Cateterización del bulbo yugular en niños con traumatismo encefálico grave
}

\author{
Cristián Clavería R.'; Alejandro Donoso F.'; Cristián Valverde G.'; \\ Bernd Oberpaur W.'; Mario Vildoso F.'; Alejandro Gallardo F.' ; Patricia Frangini S.?
}

\begin{abstract}
Resumen
Objerivo: destccar to utiidad de la medición de la saluración de oxigerio en la songre venosa del bulbo vugular

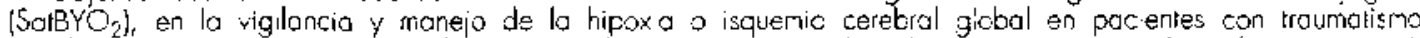
encéfaloc aneano grove y sus correlcciores con las presiones ar erial medip. intracioneono y de peftusión cereoral. Pacientes y méfodos. once niños IFromedio de edaces 98 meses, nueve varonesì, con traumalismo croneoencefálico grave |cclificación de Glasgowigual o nenor a 8). causado por impacio con vehiculos en lo vía pittica, fueron admitidos a una unided de cuidados i lensivos pediátricos de un hospi:al suburbano óe Santiago duranle un periodo de 12 meses. En rodos se introdujo un coleter en el bulbo yugular y se hiciercn mediciones de SolBYO 2 . Resultados: la SciBYOz era anormalmente baja en cuatro pacientes, er lódos habio acentuada hiperventilación y en dos hipertensićn intracroneana. wero se normclizó mediarite ajusles en los conlioles de los ventiledores mecónicos y el manejo efectivo de a hipertensićn intracraneana. No se regislaron complicaciones debidas cl orocedimienio. Todos los pocientes soprevivieron. Conclusiones; el mélodo es aolicable y seguro en ocierles de unidades pediálricas de

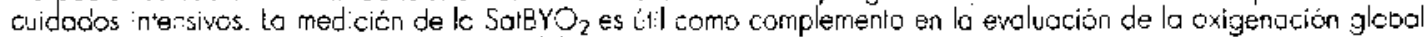
cerebrol en pocientes con trau matismo encétclucroneanio grave.
\end{abstract}

Palabras clave: caleterización bulbo yugular, saluración de oxigeno bulbo yugular, rroumatismo encélalocraneano. TEC. pedialric. ${ }^{\text {i }}$

\section{Yugular bulb blood oxygen saturation in children with severe head trauma}

Obiective: to -ighlight usefulness of vugular bult tilood cxygen saluration 'SalBYO $\mathrm{O}_{2}$ l in the mancgement of children with severe heod trauma and to correale il with introngrecl pressure, ce'ebral pertusion pressure and mean arterial pressure. Potients and methods: vugular bulb was catheterized cnc blood samples were obtained for SalBYO. in all elever chidren (9 moles) admited to a pedicir $c$ intersive ccre urit of c suburbar, gereral hospilal at Sanliago, Chile, olong o 12-month period, becouse of severe head roumc imecn Gkosgow score: 7i caused by motor vehicle injuries. mean ege of patients was 98 menttis. Resuits abnermaliy low SatBYO- was recorded in four polients it was asssciated to severe hyperventilation in al four and with increosed intracraneal pressure in two, retwrning to normol ater proper adjustment of mechanical vertilation set.ngs and treatment of intracraneal hyperterision. No complications d.Je :c the use of the catheler were obseved, neither deahs ocured in this series Conctusion: vugular butb

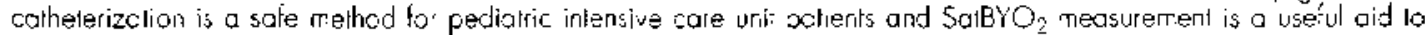
evoltale glota: cerebral oxygenotion in children with severe head raurra.

(Key words: yugular bulb cotheterization, yugular bulb blood oxygen saturation, heod laumo. pedialrics.)

En Chile los traunatismos y envenenamientos son la tercera causa de muerte en la pobla-

1. Unidad de Cuidados Intensivos Pediátricos. Hospital Dr. Sótero del Río.

2. Incerna Medicina. Pontificia Universidad Catolica de Chile. ción general, siendo el traumatismo encéfalocraneano (TEC) la primera causa de mortalidad en el grupo etáreo comprendido entre los 5 a 14 aก̃os ${ }^{1-3}$.

En los pacientes que han sufrido TEC se observa que posterior al daño primario se pueden presentar trastornos secundarios, como hipoten- 
sión arterial, hipoxia, anemia, convulsiones y fiebre, los cuales pueden llegar a comprometer tanto el flujo sanguíneo cerebral (FSC) como el metabolismo cerebral, con un claro efecto deletéreo sobre neuronas potencialmente viables, fenómeno denominado daño secundario ${ }^{4 \cdot 6}$. En la última década, el énfasis del manejo médico de los pacientes con TEC se ha centrado en la prevención del daño cerebral secundario. el cual es consecuencia de una inapropiada entrega de oxígeno.

Dentro de los factores que determinan una inadecuada entrega de oxígeno destaca la isquemia cerebral como consecuencia de hipoxemia arterial, anemia grave o una reducción crítica de la presión de perfusión cerebral (PPC). Esta última corresponde a la diferencia entre presión arterial media (PAM) y la presión intracraneana (PIC), por lo que un descenso de la presión arterial a elevación de la presion intracraneana determinan una caída de la $\mathrm{PPC}^{7-8}$

En la gran mayoría de los pacientes que fallecen por TEC grave $(90 \%)$, hay evidencias neuropatológicas de isquemia cerebrat ${ }^{9} 10$.

En la realidad clínica habitual, las variables más frecuentemente controladas en los pacientes con TEC grave son la PIC y la PAM, desde las cuales se infiere la PPC. Las decisiones de tratamiento se basan fundamental y casi exclusivamente en estas variables. La literatura internacional ha destacado la utilidad de la monitorización de la oxigenación cerebral por medio de la medición de la saturación venosa de oxígeno a nivel del bulbo yugular $\left(S a t B Y O_{2}\right)$. El valor clínico de la $\mathrm{SatBYO}$ se basa en su capacidad de reflejar el estado de equilibrio entre los requerimientos metabólicos y la entrega de oxígeno a nivel cerebral ${ }^{11-17}$.

El objetivo del presente estudio fue realizar un análisis de la información obtenida de la monitorización de la oxigenación cerebral por medio de la medición de la SatBYO $\mathrm{S}_{2}$ en los pacientes que han sufrido un TEC grave, incluyendo dentro del análisis la correlación existente entre la $\mathrm{SatBYO} \mathrm{B}_{2}$ con los valores de PIC, PPC y parámetros hemodinámicos y ventilatorios presentados por los pacientes durante su evolucion.

\section{Pacientes y métodos}

Durante un perfodo de doce meses, entre marzo de 1996 y ınarzo de 1997, todos los pacientes con diagnóstico de ingreso de TEC grave (Escala de conia de Glasgow $\leq$ 8), fueron enrolados en el presente estudio, previo conocimiento y autorización por escrito por parte de los padres o familiares del paciente. Todos los pacientes fueron in. gresados, monitorizados y tratados en la Unidad de Cuidados Intensivos Pediátricos del Hospital Dr. Sótero del Río.

En cada uno de los pacientes estudiados se consideraron datos como: edad, peso, sexo, diagnóstico asociado, PRISM (Pediatric Risk Score of Mortality) y ROM (Risk of Mortality $)^{18}$. Por su parte. de la técnica misma de instalación del catéter de BY: momento de instalación. compliçaciones en la inscrción. tiempo de permanencia del catéter, complicaciones. evolución y mortalidad del grupo

Se realizó tolnografía axial computadorizada de cerebro sin medio de contraste en todos los pacientes dentro de las primeras seis horas de haber ingresado. Las lesiones intracraneanas que deteminaron ocupación de espacio fueron evacuadas quirúrgicamente.

Se efectuó un registro continuo invasivo de la presión arterial (PAS, PAD, PAM), presión intracroneana (PJC). presion venosa central (PVC), saturación arterial de oxigeno $\left(\right.$ Satart $\mathrm{O}_{2}$ ) y capnografía $\left(\mathrm{EtCO}_{2}\right)$. La medición de la PIC se realizó por medio de uno de los siguientes dispositivos: captador subdural, captador intraparenquimatoso (Codman (8) o captador intraventricular.

La evaluación de laboratorio incluyo: medición de saturación venosa de oxígeno del bulbo yugular (SatBYO), análisis de gases arteriales y hematocrito. Todos estos criterios fueron controlados cada 8 horas desule su instalación, durante las primeras 36 h de evolución. Si un valor de SatBYO, se consideraba patológico, se repetía la evaluación luego de establecer las medidas terapéuticas indicadas.

La inserción del catéter en el bulbo yugular fue responsabilidad del médico residente de $\mathrm{UCl}$, siendo ef acceso a la vena yugular intema derecha por aproximación medial percutánea a nivel del cartilago cricoides, tomando como puntos de referencia el latido de la arteria carótida por dentro y el ángulo superior del espacio supraclavicular determinados por los fascículos del músculo esternoclei domasloideo al insertarse en la clavicula. La ubicación de la cabeza es en ligera extenșion y en posición neutra. Utilizando la lécnica de Seldinger se introduce en forma retro. grada un catéter Arrow International@. Ga $\mathrm{N}^{\circ} 200 \mathrm{Ga}$ $N^{\circ} 18$ (dependiendo de la edad del paciente) dentro de la vena yugular interna en dirección al foramen magnum. hasta el punto en el cual se aprecia una resistencia. procediendo a retirarlo le vemente y aspirando sangre, con lo que se comprueba su permeabilidad ${ }^{12}$. Posteriomente se procede a determinar su adecuada posición a través de una radiografía laterol de cráneo, tomando como puntos de ré ferencia una línea recra que se extiende desde el espacio articular atlanto-occipital hasta el borke inferior del reborde orbitario. Es aceptado como ubicación adecuada cuando la punta del catéter se localiza hasta $2 \mathrm{~cm}$ por debajo de dicha línea, asegurándose así evitar la contaminación sanguínea extracerebral, lo que podría alterar la medición efectuada 11.14.

Se mantuvo el catéter permeable con solución salina al $0,9 \%$ heparinizada (J $\mathrm{U} / \mathrm{ml}$ ) y este fue destinado únicamente a ba toma de muestra de sangre para medición de la saturación venosa de oxígeno. 
Las mediciones para oxigenación global y cerebral realizadas fueron contenido arterial de oxígeno $\left(\mathrm{CaO}_{2}\right)$, contenido venoso de oxigeno BY $\left(\mathrm{CBY} \mathrm{O}_{2}\right)$. diferencia arteriovenosa yugular de $\mathrm{O}_{2}\left(\triangle \mathrm{ABYO} \mathrm{O}_{2}\right)$, saturación arterial $\mathrm{de} \mathrm{O}_{2}$ $\left(\mathrm{Sat}_{a} \mathrm{arO}_{2}\right)(\mathrm{VR}=93,9 \pm 1,0 \%)$ y saturación venosa $\mathrm{BY}$ de $\mathrm{O}_{2}$ (Sat $\mathrm{BYO}_{2}$ ) (VR $=61,8 \pm 3,7 \%$ ) (tabla 1 ). Los valores de reterencia (VR) utilizados corresponden a los de adultos jóvenes sanos en reposo 11 . zll.

Se consideró situación límite, determinante de hipoxia 0 isquernia cerebral, valores de Sat $\mathrm{BYO}_{2}<55 \% 11.21$.

El protocolo terapéutico estuvo orientado principalmente a la prevención del daño cerebral secundario $c$ incluyó medidas antiedema cerebral con elevación de la cabeza a $30^{\circ}$. cabeza en posición nentra y evitando todo tipo de estímulos auditivos, visuales y maniobras dolorosas en el paciente: ventilación mecánica manteniendo $\mathrm{PaCO}_{2}$ entre 30 y $35 \mathrm{mmHg}$ : aportes hídricos según sus requerimientos basales más aportes de volumen exira en búsqueda de euvolemia; uso de terapia anticonvulsivante profiláctico o terapéutica en base a fenobarbital con dosis de carga (20 $\mathrm{mg} / \mathrm{kg} /$ dia) a midazolarn en infusión continua $(0,1-0,4$ $\mathrm{mg} / \mathrm{kg} / \mathrm{h}$ ): sedo-analgesia con infusión continua de mida. zolam y fentany] (1-2 $\mu \mathrm{g} / \mathrm{kg} / \mathrm{h})$. Cuando los pacientes presentaron ascenso mantenido de la $\mathrm{PIC}>15 \mathrm{mmHg}$, por 60 segundos o más, fueron tratados con uso secuencial de drenaje de LCR de ser esto posible, hiperventilación para $\mathrm{EtCO}$, entre 25 y $30 \mathrm{mmHg}$, manicol $0,25 \mathrm{~g} / \mathrm{kg}$ peso, administrado en bolo y en 20 minutos, o uso de barbilüricos (tiopental 2-3 $\mathrm{mg} / \mathrm{kg}$ de peso). En caso de persistir la PJC elevada. se efectuó evaluaeión con TAC de cerebro para determinar la causa de hipertensión endocraneana y la necesidad de una eventual intervención neuroquirürgica. En relación a la medición de la PPC se consideró como valor adecuado sobre $60 \mathrm{~mm} \mathrm{Hg}$. los valores inferiores se optimizaron en base a la nontualización de la PIC cuando correspondia o elevación de la PAM por medio del aporte de volumen o uso de drogas vasoactivas.

Todo catéter al momento de su retiro fue enviado para su cultivo según la norma utilizado en nuestro centro asistencial.

\section{Análisis estadístico}

Se efectuó un estudio de estadística descriptiva para los aspectos demográficos de la población y del procedimiento, obteniéndose los valores de tendencia central y dispersión como promedio $(\overline{\mathrm{x}})$ y desviación estándar (DE). Se realizó un estudio de sensibilidad y especificidad para evaluar los cambios de la $\mathrm{SatBYO}_{2}$ en relación a los parámetros clínicos estudiados $\left(\mathrm{PaCO}_{2}\right.$, PIC y PPC).

\section{Resultados}

Durante el período descrito se procedió a la cateterización del BY en once pacientes, nueve

\section{Tabla 1}

\section{Parámetros de oxigenación global y cerebral evaluados}

Contenido arterial de oxigeno $\left(\mathrm{CaO}_{2}\right)$ :

$\mathrm{Hb} \times 1,34 \times \mathrm{Sat} \operatorname{artO}_{2}+0,003 \times \mathrm{PaO}_{2}(\mathrm{VR}=19,6 \pm 1,2 \mathrm{vol} \%)$

Contenido venoso de oxígeno $\mathrm{BY}\left(\mathrm{CBYO}_{2}\right)$ :

$\mathrm{Hb} \times 1.34 \times \mathrm{SatBYO}+0,003 \times \mathrm{PBYO}_{2}(\mathrm{VR}=12,9=1,3$ ×ol\% $)$

Diferencia arterio-venosa yugular de $\mathrm{O}_{2}(\triangle \mathrm{aBYO})$ :

$\left(\mathrm{CaO}_{2}-\mathrm{CBYO}_{2}(\mathrm{VR}=6,7 \pm 0,8 \mathrm{rol} \%)\right.$

Saturación arterial de $\mathrm{O}_{2}$ (Sat artO $\mathrm{O}_{2}$ ) ( $\left.\mathrm{VR}=93,9 \pm 1,0 \%\right)$

Saturación venosa $\mathrm{BY}$ de $\mathrm{O}_{2}\left(\mathrm{Sat} \mathrm{BYO}_{2}\right)(\mathrm{VR}=61,8 \pm 3,7 \%)$

de sexo masculino, con un promedio de edad de 8 años 2 meses (rango 1 año 3 meses -13 años). La causa del TEC fue atropello por automóvil en todos los pacientes. El diagnóstico asociado más frecuente fue el politraumatismo $(9 / 11)$. El Glasgow de ingreso a la Unidad de Energencia fue en promedio de 7 puntos (rango 5 - 8 puntos). El PRISM fue en promedio de 16,4 $\pm 6,2$, con un ROM $17 \pm 15,32 \%$. La tomografía axial computadorizada de cráneo evidenció principalmente contusiones hemorrágicas $(6 / 11)$ (tabla 2). Permanecieron en ventilación mecánica en modalidad presión control, en promedio 6 días (rango 2-9 días). La monitorización de la PIC se realizó por medio de un captor subdural ( 8 pacientes), captor intraventricular ( 2 pacientes) y captor intraparenquimatoso (2 pacientes); en un paciente se instalaron 2 catéteres. El momento de la instalación fue en promedio a las 7 horas desde e] ingreso (rango $1-24$ horas) con un tiempo de permanencia de $4,2 \pm 2,4$ dias. La instalación del catéter del BY se realizó en promedio a las 8,6 horas desde su ingreso (rango 1 20 horas), con un tiempo de permanencia de 3,8 $\pm 0,9$ días. No hubo complicaciones en relación a la técnica, como tampoco modificación significativa de la PIC durante el procedimiento de instalación.

En todos los pacientes se midió la SatBYO al menos en tres oportunidades, con un total de 37 mediciones para el grupo. En aquellos casos en que se obtuvo un valor considerado como patológico ( $\mathrm{SatBYO} \mathrm{YO}_{2}<55 \%$ ), se repitió el procedimiento con el fin de evaluar las medidas 
Tabla 2

Características de los pacientes con bulbo yugular

\begin{tabular}{|c|c|c|c|c|c|c|c|}
\hline Caso & $\begin{array}{l}\text { Edlad } \\
\text { (años) }\end{array}$ & Sexo & Grasgow & PIC & PRISL/ROM & TAC & Sobreviye \\
\hline $\mathbf{I}$ & 12 & $M$ & 5 & SD & $21 / 24$ & Daño difuso & Sí \\
\hline 2 & 13 & $\mathbf{F}$ & 7 & \$D/lV & $28 / 55$ & $\begin{array}{l}\text { Contusiones + } \\
\text { hidrocefalia ag. }\end{array}$ & Sí \\
\hline 3 & 11 & $M$ & 6 & SD & $23 / 33,6$ & $\begin{array}{l}\text { Contusiones + } \\
\text { H. sub y epidural }\end{array}$ & Sí \\
\hline 4 & 1,25 & $M$ & 8 & IV & $5 / 2,1$ & Hemorragia subracnoidea & Sí \\
\hline 5 & 3,9 & M & 8 & SD & $13 / 9,4$ & Contusiones & Sí \\
\hline 6 & 13 & $\mathbf{M}$ & 8 & SD & $19 / 16,4$ & $\begin{array}{l}\text { Dafio difuso }+ \\
\text { henorragia subaracnoidea }\end{array}$ & Sí \\
\hline 7 & 10 & M & 8 & SD & $16 / 11,2$ & Contusiones & Sí \\
\hline 8 & 9 & M & 8 & SD & $15 / 9,8$ & Daño difuso $+\mathrm{H}$. subdural & Sí \\
\hline 9 & 1,5 & $\mathbf{F}$ & 8 & SD & $13 / 10,2$ & Contusiones & Sí \\
\hline 10 & 4,5 & M & 5 & PAR & $13 / 8.6$ & Contustones & Sí \\
\hline ] J & 1] & M & 8 & PAR & $14 / 7,2$ & Hemorragia subracnoidea & Sí \\
\hline
\end{tabular}

F: femenino $\mathrm{M}$ : masculino $\mathrm{H}$ : hematoma

terapéuticas instauradas tendientes a su normalización.

Cuatro pacientes presentaron durante su evolución caída de la $\mathrm{SatBYO}_{2}<55 \%$, la cual se asocio con alza de PIC $>30 \mathrm{mmHg}$ en dos. El estudio estadístico demostró una sensibilidad de $50 \%$ y especificidad de $94 \%$ para $\mathrm{SatBYO}$ $<55 \%$. Asimismo, el hatlazgo de hiperventilación $\left(\mathrm{PaCO}_{2}<28 \mathrm{mmHg}\right)$ se encontro en los cuatro pacientes (sensibilidad $28 \%$ y especificidad $100 \%$ ). En todos los pacientes se normalizaron los valores de SatBYO $\mathrm{Y}_{2}$ en base a optimización de la ventilación mecánica con $\mathrm{PaCO}_{2}$ en rangos 32 a $35 \mathrm{mmHg}$ y tratamiento según protocolo de hipertensión endocraneana.

En relación a la PPC observamos valores menores de $60 \mathrm{mmHg}$ en $18 / 37$ mediciones (50\%) (rango $34-59 \mathrm{~mm} H g$ ), en cuatro oportunidades esta se asoció a SatBYO $\mathrm{O}_{2}<55 \%$ (sensibilidad de $15 \%$ y especificidad de $94 \%$ ) y en diez con PIC $>15 \mathrm{mmHg}$. En todos su normalizó la SatBYO en base a medidas terapéuticas protocolizadas: disminución de la PJC u optimización de la PAM.

La estadía en la UCI fue de 8 días en promedio (rango 4-14 días) y el de hospitalización total fuc 31 días en promedio (rango 9-60 días).
No observamos complicaciones en relación a la manténción del catêter y los cultivos de la punta de este, tomados a su retiro, fueron informados como negativos.

Ningún paciente falleció, encontrándose todos en buenas condiciones clínicas en un seguimiento de hasta 20 meses.

\section{Comentario}

En esta experiencia la cateterización del bulbo yugular fue realizada en pacientes que sufrieron TEC grave con Glasgow $\leq 8$ puntos, una vez ingresados a la $\mathrm{UCl}$, previamente evaluados con TAC de cerebro $y$ habiendo resuelto quirúrgicamente, cuando correspondía, las lesiones que determinaban un conflicto de espacio. Todos los pacientes se encontraban adecuadamente monitorizados, lo que permitió evaluar su situación hemodinámica global y cerebral por medio de la medición de la PIC y cálculo de la PPC. con el fin de delectar y tratar en forma oportuna situaciones clínicas que pudiesen condicionar hipoxia o isquemia cerebral (insultos secundarios). 
En relación a la información obtenida del estudio de las variables fisiológicas y teniendo en consideración el número de pacientes analizados, podemos comentar que no observamos correlación entre elevaciones de la PIC y caídas en la $\mathrm{SatBYO} \mathrm{O}_{2}$ lo cual podría explicarse por la naturaleza global de la medición aportada por el bulbo yugular ", como también por to oportuno y eficaz de las medidas terapéuticas empleadas, evitando de esta manera que el alza de la PIC se tradujeráa en caídas patológicas de la $\mathrm{SatBYO}$. Desde otro punto de vista, la disminución de SatBYO $\mathrm{B}_{2}$ sería más tardía que los ascensos de la PIC. Observamos una correlación directa entre el nivel de hiperventilación y las caídas patologicas de la SatBYO $\mathrm{Y}_{2}$, normalizándose esta una vez. optimizada la ventilación, incrementando los valores de la $\mathrm{PaCO}_{2}$. La hiperventilación se utiliza rutinariamente en el tratamiento de los pacientes con sospecha de aumento de la $\mathrm{PIC}^{22}$. La reducción aguda en la presión arterial de dióxido de carbono $\left(\mathrm{PaCO}_{2}\right)$ produce vasoconstricción cerebrovascular y una caída en el $\mathrm{FSC}^{23}-2.5$. El volumen sanguíneo cerebral subsecuentemente cae, determinando una reducción en el volumen intracraneal y una rápida caída en la PIC. Sin embargo, el uso agudo de la hiperventilación dentro de las primeras 24 horas posterior a un TEC grave presenta un riesgo potencial de isquemia cerebral ${ }^{25}, 26$. La hiperventilación agresiva produce una marcada reducción en el FSC, lo cual puede determinar un aumento o exacerbación de la isquemia cerebral, con el consecuente riesgo de daño secundario $6,17,22,25,26$. Esto refuerza la utilidad de la medición de la $\mathrm{SatBYO}_{2}$ en la supervisión clínica de la estrategia terapéutica empleada en los pacientes con TEC grave.

Se ha descrito en forma teórica la relación proporcional existente entre la PPC y el flujo sanguíneo cerebral, postulándose que presiones de perfusión elevadas serían un indicador de la adecuada hemodinamia cerebral en pacientes con trauma cerebral ${ }^{27,} 28$. Sin embargo otros estudios, en que midieron PPC y SatBYO, no demostraron el enunciado previo ${ }^{27-29}$.

La obtención de sangre venosa desde el BY permite medir la $\mathrm{SatBYO}_{2}$ y así calcular el $\mathrm{CBYO}_{2}$ y la $\triangle \mathrm{aBYO}$ adquiriendo de esta forma información indirecta del flujo sanguíneo cerebral global y del metabolismo cerebral. La disminución en la $\mathrm{SarBYO}_{2}$ sería un indicador del incremento en la extracción de oxígeno del cerebro para mantener así cubiertas sus necesidades metabólicas ${ }^{15,30,31}$. Cuando este mecanismo de compensación es sobrepasado se produce una situación de hipoxia cerebral oligohémica con el consiguiente daño neurológico secundario $15,30,31$.

Una de las mayores limitaciones de la medjción de la SatBYO ${ }_{2}$ en detectar hipoxia o isquemia cerebral son sus restricciones anatómicas. La medición de la SatBYO $\mathrm{YO}_{2}$ corresponde a la evaluación global de la oxigenación cerebral. Situaciones de isquemia regional pueden estar presentes y pueden no ser detectadas por cambios en la SatBYO ${ }_{2}{ }^{11}$. Otra objeción importante a considerar en las mediciones de la SatBYO de nuestros pacientes es que estas fueron realizadas en forma aislada, lo cual no permite una evaluación continua. Actualmente se dispone de catéteres de fibra optica que permiten su medición continua ${ }^{14,16}$.

En consideración a las bases anatómicas de la circulación venosa cerebral, existen diversos métodos para determinar cuál vena yugular interna debe ser cateterizada. Así, en pacientes con dafio cerebral difuso, el catéter debe ser ubicado en el lado de drenaje venoso dominante, el cual es generalmente el lado derecho. Dos métodos son comúnmente utilizados para determinar cuál de las dos venas yugulares internas es la de mayor flujo o dominante. El primer método es por compresión manual secuencial de cada vena yugular interna, la compresión de la vena que produzca mayor elevación de la PIC es identificado como el lado dominante, recibiendo la mayor proporción del flujo venoso cerebral. El segundo modo es la visualización del foramen yugular de mayor tamaño en el TAC de cráneo de ingreso, asumiendo que es en este donde se encuentra el bulbo yugular dominante ${ }^{11}, 32,33$.

Si el daño cerebral es focal, algunos investigadores ubican el catéter en relación al hemisferio con mayor daño y otros sugieren su ubicación en relación a la vena yugular dominante ${ }^{11}$ 32,33 . Nosotros utilizamos en todos nuestros pacientes la vena yugular interna derecha, que correspondía ser la dominante.

Se describen algunas complicaciones en relación a la cateterización del BY que incluyen complicaciones en relación a la técnica $11,34,35$ como elevación de la PIC; punción inadvertida de la arteria carótida con sangramiento, daño de 
nervios del cuello y neumotórax; complicaciones en la mantención del catéter como trombosis, elevación de la PIC e infección, las cuales pueden ser más frecuentes cuando el catéter es utilizado con un propósito distinto a la toma de muestra de sangre venosa del BY, tales como la infusión de derivados sanguíneos, medicamentos $u$ otras soluciones ${ }^{11,34,35}$.

Las complicaciones observadas en relación al procedimiento en los pacientes pediátricos es similar a la presentada por el uso de otros catéteres vasculares invasivos. En nuestra experiencia no se observaron estas complicaciones.

Está contraindicada la técnica en aquellos pacientes que presentan lesiones de columna cervical, sospecha de trombosis venosa y en pacientes con trastorno significativo de la coagulación. La presencia de traqueostomía es una contraindicación relativa debido al potencial incremento de las infecciones ${ }^{11}$.

Considerando que la medición de la $\mathrm{Sat} \mathrm{BYO}_{2}$ es una lécnica invasiva, debe ser reservada s6lo para aquellos pacientes que presenten riesgo significativo de isquemia cerebral y en pacientes pediátricos que deban ser hiperventilados en algún momento de su evolución, como es el caso de los pacientes que han sufrido un TEC grave.

Podemos concluir que la cateterización del BY en la población pediátrica puede ser exitosa y segura, factible de realizar en las Unidades de Cuidados Intensivos Pediátricos. A través de esta técnica podemos obtener muestra de sangre venosa desde el BY, lo que permite medir la capacidad global de extracción de oxígeno del cerebro, elemento complementario de la adecuada monitorización y un método útil de evaluación de la terapia empleada en los pacientes que han sufrido un TEC graye.

\section{Referencias}

1. Instituto Nacional de Estadisticas. Anuario Demografico. Santiago, Chile, 1993: 318-336.

2. Boza C. Domoso A, Gigori.t J. et al: Epideniología del traumatismo encéfalocraneano en niños del área surorience de Santiago. Rev Chil Pediatr 1997; 68: 83-87.

3. Boza C. Donoro A, Gigotx $J$, ef al: Traumatismo en. céfalocraneano en niños del área suroriente de Santiago. Rev Chil Pediatr 1997; 68: 88.92.

4. Chesnut RM: Secondary brain insults after head injury: Clinical perspectives, New Horizons 1995; 3 : 366-375.
5. Chesnut RM, Marshall LF, Klatwer MR, ef al: The role of secondary brain injury in determining outconje from severe head injury. I Trauma 1993: 34: 216-222.

6. Chesnat RM, Marsholl $S B$, Piek J, et al: Early and late systemic hypotension as a frequent and fundamental source of cerebral ischemia following severe brain injury in the traumatic coma data bank. Acla Neurochir Suppl 1993; 59: 12 I-125.

7. Patsun OB. Strundgatard S, Edvinswon L: Ccrebral autoregulation. Cerebrovasc Brain Metab Rev 1990: 2 : 161-192.

8. Resner MJ. Danghton S: Cerebral perfusion pressure management in head injury. J Trauma 1990; 30: 9.3. 941.

9. Grahan DI, Adams JH: lschaenic brain damage in fatal head injuries. Lancet 1971: 1: 265-266.

10. Grohant DI, Ford 1, Adams JH: Ischaemic brajn damage in fatal ron-missile head injuries. J Neurol Neurosurg Psychiatry 1989; 52: 346-3.50.

11. Feldnun Z, Robertson CS: Monitoring of cerebral hemodynamics with jugular bulb catheters. Critical Care Clinics 1997: 13:51-77.

12. Andrews PJD. Deurden Nit, Miller JD: Jugular bulb cannulation: Description of a cannulation lechnique and validation of a new continuous monitor. Br $\mathrm{J}$ Anaesth 1991; 67: 553 .

13. Robertson CS. Narayan RK. Gukastan ZL, et al: Cerebral arteriovenous difference as an estimiate of cerebral blood flow in comatose patients. J Neurosurg 1989: 70: $222-230$.

14. Foriune JB, Feustel PJ, Wiegle CG et at: Continuous measurement of jugular venous oxygen saluration in response to transient elevalions of blood pressure in head-injured patients. J Neurosurg 1994; 80: 461-468.

15. Chan KH, Miller ND. Dearden NM, et at: The effect of changes in cerebral perfusion pressure upon middle cerebral artery blood flow velocity and jugular bulb venous oxygen saturaron after severe brain injury. J Neurosurg 1992; 77: 55-61.

16. Sheinherg $M, X$ unter $M J$. Robertson CS, ef ad: Continuous monitoring of yugular venous oxygen saturaron in head injured patients. J Neurosurg 1992: 76 $2 ! 2-217$

17. Gopinath SP, Roberison CS, Conrant CF, et al: Jugular venous desaturation and outcome after head injury. J Neurol Neurosurg Psychiatry 1994; 57: 717. 723.

18. Pollack MM. Ruttiman UE, Getson PR: The pediatric risk of mortality (PRISM) score. Crit Care Med 1988; 16: $1110-1116$

19. Bankier A.A. Fleischmann D, Windisch A, el al. Position of yugular oxygen saturation catheter in patients with head crauma: Assessment by use of plain films. Arnerican Journal of Radiology 1995; I64: 437.

20. Gibbs EL. Lennox WG, Gibbs FA. Arterial and cerebral venuus blood. Anterial-venous differences in man. J Biol Chem 1942; 144: 325-332.

21. Cook DJ, Oliver WC, Orstulak TA, et at: A prospective, randomized comparison of cerebral venous oxygen saturation Juring normotherinic and hypothermic cardiopulmonary bypass. J Thorac Cardiovase Surg 1994; 107: 1020-1029. 
22. Yund $K D$, and Diringer $M N$ : The use of hyperventilation and its impact on cerebral ischemio in the treatment of traumatic brain injury. Critical Cate Clinics 1997; 13: 163-184.

23. Grubb RL, Raichle ME. Eichling JO et at: The effects of changes in $\mathrm{PaCO}_{2}$ on cerebral blood volume, blood flow and mean vascular transit time. Stroke 1974; 5 : 630-6.39.

24. Konfos HA, Raper AH. Patterson JL/: Analysis of vasoactivity of local $\mathrm{pH}, \mathrm{PCO}_{2}$ and bicartonate on pial vessels. Stroke 1977: 8: 358-360.

25. Marion DW. Firlik A Mcluaghlin MR: Hyperventilation therapy for severe traumatic brain injury. New Horizons 1995; 3: 439-447.

26. Bullock $R$. Chesnat RM, Cliffon $G$, et al: The use of hyperventilation in the acute management of severe trausmatic brain injury. In: Bullock R. Chesnut RM. Clifton $G$, et al (eds): Guidelines for the management of severe head injury. New York. Brain Trauna Foundation. 1995

27. Cruz J. Jaggi $\boldsymbol{U}$. Horfistad OJ: Cerebral blood flow, vascular resistance and oxygen metabolism in acute brain trauma; Redefining the role of cercbral perfusion pressure? Cuil Care Med 1995: 23: 1412-1417.
28. Rosner $M J, D$ usghton S: Cerebral perfusion pressure management in head injury. J Trauma 1990; 30: 933941 .

29. Chan KH, Dearden NH, Miller JD. ef al: Multimodality monitoring as a guide to treatement of intracranial hypertension after severe brain injury. Neurosurgery $1993 ; 32: 547-553$.

30. Gibert Ji Estituation of CBF by cerebral venous oxygen difference. J Neurosurg 1989; 71:790-791.

31. Pautson OB. Sirandguard S. Edvinsiron L: Cerebral auturegulation. Cerebrovase Brain Melab Rev 1990; 2: 161-192.

32. Denys BG, Uretsky B: Anatomical variations of internal jugular vein location: Impuct on central venous access. Crit Care Med 1991: 19: 15!6.

33. Sroccherti $N$, Barbagallo $M$, Gordon $C R$, ef al: Cerebral venous oxygen saturation siudied using bilateral samples in the jugular veins. Neurosurgery 1994: 34: 38.

34. Goetting $M G$. Preston $G$; Jugular bulb catheterization, Experience with 123 patients. Crit Care Med 1990: 18: 1220.

35. Gaye $M O$, Frewen $T C$, ef at: Jugular venous bult catheterization in infants and children. Crit Care Med 1989; $17: 385-388$.

Esta publicación está disponible en copias de microfilms de 16 y $35 \mathrm{~mm}$ y microfichas de $105 \mathrm{~mm}$, las que pueden solicitarse a:

University Microfilms International 300 North Zeeb Road

Ann Arbor, Michigan 48106, USA.

This journal is also available in $16 \mathrm{~mm}$ microfilm, $35 \mathrm{~mm}$ microfilm and $105 \mathrm{~mm}$ microfilm copies through

University Microfilms International,

300 North Zeeb Road,

Ann Arbor, Michigan 48106, USA. 